\title{
Predictive Factors of Major Lower Extremity Amputations in Diabetic Foot Infection: A Cross-sectional Study in Three District Hospitals
}

Kow Ren $\mathrm{Yi}^{1}$

${ }^{1}$ Department of Orthopaedics, Hospital Tengku Ampuan Afzan

Presenter: Kow Ren Yi

Introduction: Limb amputation in diabetic patients is associated with severe economic, social and psychological effects on patients and their families. This study aimed to evaluate the predictive factors of major lower limb amputation among patients with diabetes mellitus. Materials and Methods: This cross sectional study involved patients from a cluster of three district hospitals in Pahang, Malaysia (Hospital Kuala Lipis, Hospital Raub, and Hospital Bentong) from the $1^{\text {st }}$ September 2014 to the $31^{\text {st }}$ December 2015. Age, sex, ethiniticy, facilities, presence of hypertension, duration of illness, fever, haemoglobin level, white blood cell level, urea, creatinine, history of more than three limb salvaging surgeries, diagnosis and tissue culture were assessed to determine the predictive factors of major lower limb amputation. Results: History of more than three times limb salvaging surgeries, white blood cell count more than $15 \times 10^{9} / \mathrm{L}$ and longer duration of disease were significant predictive factors of major lower limb amputations in patients with diabetic foot infections. Conclusion: This study highlights the importance of initial limb salvaging surgeries as history of more than three times limb salvaging surgeries is a predictive factors of major amputation in diabetic foot infection. 